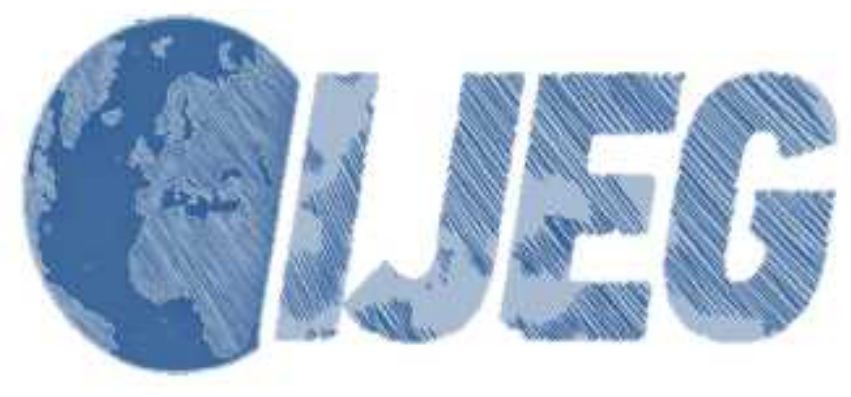

International Journal of Engineering and Geosciences (IJEG), Vol;2, Issue;01, pp. 17-26, February, 2017, ISSN 2548-0960, Turkey, DOI: $\underline{\text { 10.26833/ijeg.286691 }}$

\title{
OPEN GEOSPATIAL CONSORTIUM WEB MAP AND FEATURE SERVICES AND FREE/OPEN SOURCE SERVER/CLIENT SOFTWARES
}

\author{
Varol, M.., ${ }^{1 *}$ Sanloglu, I., ${ }^{2}$
}

${ }^{1}$ General Command of Mapping, Cebeci, Ankara, (mesud.varol@gmail.com)

${ }^{2}$ Assist.Prof. Dr., Selçuk University, Geomatics Engineering, Konya, (sanlioglu@ selcuk.edu.tr)

"Corresponding Author, Received: 20/01/2017, Accepted: 31/01/2017

\begin{abstract}
Usage of geospatial data enables decisions to be more effective and stronger in critical fields. Since geospatial data is very expensive source and the most time-consuming step, mostly it is not possible to find the required data ready to use. Therefore different projects have been developed and tried to implement by several countries to keep geospatial data which is required to collect and managed, accessible and usable. At this study Open Geospatial Consortium standarts were explained, the focus was given on WFS and WMS because of their increasing popularity. Concurrently free and/or open source WMS/WFS server and client softwares were investigated.
\end{abstract}

Keywords: Interoperability, Standards, Open Geospatial Consortium, Web Map Sevice, Web Feature Service, Open Source Softwares. 


\section{INTRODUCTION}

Gathering, compiling, presenting and analyzing of the information is one of the most sought-after topics to be explored today. If the positional characteristic of the knowledge that many disciplines need in common also belongs to the account, the degree of complexity will increase in the same way. It is also important that the information which is no doubt about its authenticity and proven knowledge needs to be communicated to the right place at the right time, fast and up-to-date. Developments in computer technology have increased the feasibility of issues such as data storage, data organizing, data sharing, re-evaluation of data, data analysis and the best representation of the earth's surface. The diversity of applications has increased as the importance of geographical information systems has increased and many disciplines have begun to benefit from this technology. The variety of applications and the geographic data structures used in applications has created the needs to regulate interoperability, data and application standards. For this purpose, the international standards used in the internet based geographical information systems have been examined and the developed application has been ensured to conform to the mentioned standards.

In the first part of the done work, the definition of interoperability, its importance and the stages in which it can be carried out today, the advantages of these standards and the aims of their development are explained from the standards established to enable interoperability. In the second part, OGC standards are discussed. In the third part, WMS and WFS, which are frequently used OGC standards, are discussed in more detail. The fourth section introduces open source software that will allow the server to be installed and then be used by the client to view the data. In the last section, the facilities provided by WFS for data presentation are mentioned in terms of interoperability and evaluated in terms of geomatics engineering.

\section{INTEROPERABILITY AND FUNDAMENTAL CONCEPTS}

In order to fully understand and use the capabilities and benefits of information system technologies, the data must be shared between different users, and the interoperability of the systems must also be ensured.

The broadest definition of interoperability, which can be described as the ability of information to be used and transferred between institutions and information systems, is effective information sharing (Anonim, 2012).

Interoperability; is also referred as "the ability of a system or process to use its knowledge and / or functions of another system or process within the framework of common standards" (Europa, 2016).

Interoperability needs can be examined in three dimensions as technical, organizational and semantic. At technical level, the organizational dimension is based on engineering methodologies such as process modeling languages, object oriented software engineering, rather than technology, while focusing on the technologies that enable information sharing among different applications. Within the scope of organizational interoperability, the business processes of the institutions are dealt with by modeling them in relation to the other related institutions and the business processes formed in order to provide the integration between the aims of the institutions and the applications and systems that form the technical infrastructure, in other words, to exchange the shared information more effectively and institutional structuring is targeted. It also includes processes such as re-engineering, workflow management within the organization and between institutions, and identification of needs for processes and services. In the context of meaningful interoperability, there are studies to be understood and interpreted correctly by institutions outside the institution that produces it.

There are needs to establish standards that can be applied to make interoperability possible and to use these standards.

Standards-compliant interfaces need to be created to share data. However, in addition to this compatibility, modeling, naming and identification of data and preparation of metadata forms need to be carried out in coordination. In this respect, studies are being continued in many countries and standards are being prepared by international organizations. OGC and ISO / TC 211 Geographic Information / Geomatics working groups, which have been operating since 1994, are at the top of these organizations. Their main purpose is to ensure that the geoprocessing services and the geographic data are in a certain standard. Thus establishing global international standards to ensure data exchange and interoperability (Anonim, 2016 ISO/TC, 2016).

In addition to these organizations, there are also DGIWG (Defense Geospatial Information Working Group), INSPIRE organizations.

The common aim of all these organizations is,

- Increasing access to quality geospatial data,

- Increasing the availability of current geospatial data,

- Increasing the understandability of the existing geospatial data,

- To increase the effective and economical use of geospatial data,

- Providing a common solution to the problems,

- Providing software and hardware compatibility,

- Reduction of inefficiency in the collection, storage, processing and distribution of spatial data.

\section{OGC}

OGC is an international service providing co-operation in geographical and location based studies. OGC is an international industry consortium of over 521 companies, government agencies and universities participating in a consensus process to develop publicly available interface standards. 
Latterly, with the use of the internet environment for geospatial data sharing, geographical web services has emerged. For this purpose, OGC especially aims to standardize geospatial web services by making standards and disseminate usage, and to provide interoperability for GIS (Emem O., 2007).

The mission of the OGC is to promote the development and use of advanced open systems standards and techniques in the area of geoprocessing and related information technologies. OGC's goal is to provide interoperable solutions for geographic information communities. The OGC is also known as the Open Geospatial Interoperability Specifications Consortium (OpenGIS) consortium.

As a result of OGC's ongoing studies, it is possible to use WFS, WMS, Geography Markup Language (GML), OGC Keyhole Markup Language (KML), Web Coverage Service (WCS), Web Catalog Service (CS/W), Web Map Context (WMC), Coordinate Transformation Service (CTS), Location Service and Web Registry Service (WRS).

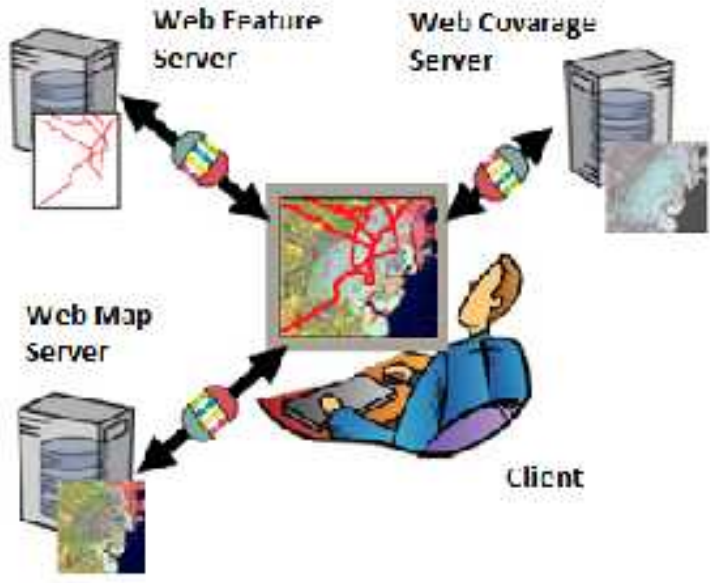

Figure 1. Server/Client schema

The OGC lists the benefits of establishing standards in the GIS field and the facilities that the generated data will have:

- Geospatial content should be easy to find, without regard to its physical location.

- Once found, geospatial content (and services) should be easy to access or acquire.

- Geospatial content from different sources should be easy to integrate, combine, or use in spatial analyses, even when sources contain dissimilar types of data (raster, vector, coverage, etc.) or data with disparate feature-name schemas.

- Geospatial content from different sources should be easy to register, superimpose, and render for display.

- Special displays and visualizations, for specific audiences and purposes, should be easy to generate, even when many sources and types of content are involved.

- It should be easy, without expensive integration efforts, to incorporate into enterprise information systems geoprocessing resources from many software and content providers (Kantar F., 2003).
OGC Web Service standards, which have a significant place in terms of web based geographic information systems are:

- Web Map Service (WMS)

- Web Feature Service (WFS)

- Web Coverage Service (WCS)

- Catalogue Service for the Web (CS/W)

- Web Coordinate Transformation Service (WCTS)

- Geography Markup Language (GML)

- Filter Encoding Implementation Specifaction (FES)

- Gazetteer Service Profile (WFS - G)

- Web Map Tile Service - WMTS

- Web Map Context (WMC)

- Web Coverage Processing Service (WCPS)

- Coordinate Transformation Service (CTS)

- Geospatial eXtensible Access Control Markup Language (GeoXACML)

- Keyhole Markup Language (KML)

- Table Joining Service (TJS)

\section{WEB MAP SERVICE - WMS}

By far the most popular and widely implemented of the geospatial standards, the OGC Web Map Service (WMS versions 1.1.1 and 1.3; ISO 19128) supports the request and display of maps derived from data accessed by the service. Maps, delivered as graphical images (GIF, JPEG, TIFF, etc.), may be requested from one or more WMSs overlaid in browsers or client applications. Features "behind" the map can also be queried, and their properties can be returned to a requesting client (Nebert D., 2007).

Schemas for validating the "capabilities" of an XML file returned from a WMS service exist, and compliance testing is available through the OGC for assessing WMS performance on all key functionalities.

WMS version 1.1.1 is the most widely deployed (ISO 19128, however, is harmonized with WMS version 1.3 but is not yet widely deployed) and is recommended for inclusion in the SDI 1.0 standards suite (Nebert D., 2007). The Geographic Data Infrastructure mentioned here is a project that is aimed at establishing the standards required for the systems used to gain the ability to use the knowledge and/or functions of another system within the framework of common standards (KYM-75, 2016).

The WMS specification standardizes simple query operations such as client requesting maps over the Internet, presenting data to servers, and displaying coordinates and attributes of requests. OGC first released the version 1.0.0 of WMS specification in 2000. After the release of the first version, the current version 1.3.0 was released in 2004 (Emem O., 2007). WMS is an international application standard published as ISO 19128 (Web Map Server Interface) standard, which was adopted by ISO in 2005 as it is OGC specification. The WMS specification defines a number of request types, and for each of them a set of query parameters and associated behaviors. A WMScompliant server must be able to handle at least the following 2 types of WMS requests: 
- GetCapabilities: is used to obtain the service metadata and accepted request parameters. Returns an XML document with metadata of the Web Map Server's information

- GetMap: is used to obtain the coordinate spatial data image. Returns an image of a map according to the user's needs.

And support for the following types is optional:

- GetFeatureInfo: A function that provides attributes for a specific detail shown in the map. Returns info about feature at a query location.

- DescribeLayer: A function that provides access to additional information about WFS and WCS layers. Returns an XML description of one or more map layers.

- GetLegendGraphic: A function that allows access to the legend created for the map. Returns a legend image (icon) for the requested layer, with label(s).

Performing mandatory and optional functions from WMS using a standard web browser is possible by requesting in URL form. The client first needs to get GetCapabilities and get the data and related parameters on the server. As a result of the GetCapabilities request, the server sends an XML file containing the necessary information (Figure 2).

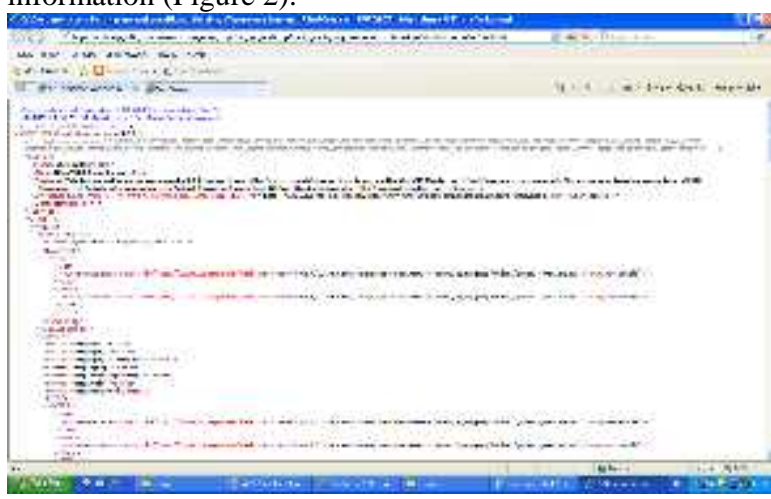

Figure 2. GetCapabilities request response

When performing a GetMap request, the client can determine the information that wants to appear on the map. A standard web browser with URL-formatted GetMap request from WMS example could look like as follows:

http://localhost/cgi-bin/mapserv.exe? map=/ms4w/apps/ms_turkiye/service/config.map $\&$ version $=1.1 .1 \&$ service $=$ WMS

\&request $=$ GetMap

\&srs=EPSG:4326

\&bbox $=-180,-90,180,90$

$\&$ format=image/png

\&layers=land_shallow_topo_2048,nehirler

\&styles=,

$\&$ transparent=true

\&width $=500 \&$ height $=300$

As a result of this request, the server from the server will return a two-layered png format image (Figure 3).

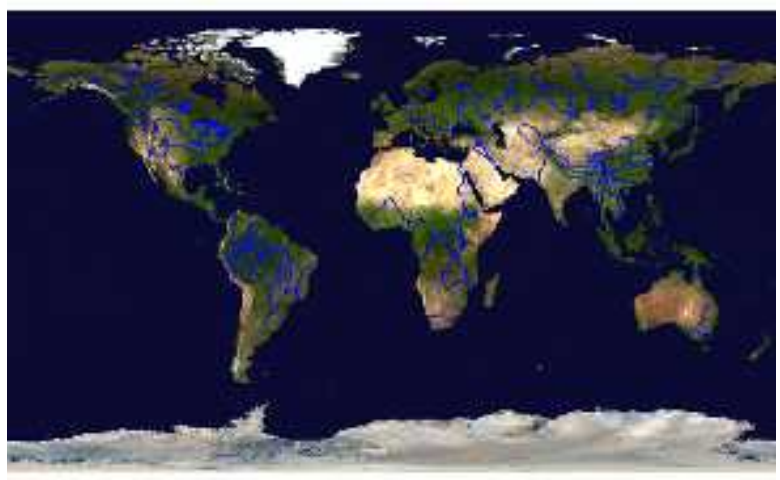

Figure 3. WMS GetMap request response

The commands used in the request are described in Table 1.

Table 1. Commands and functions in GetMap request line

\begin{tabular}{|c|c|}
\hline Command & Function \\
\hline Map & $\begin{array}{l}\text { The root layer in the WMS context } \\
\text { corresponds to the whole mapfile. }\end{array}$ \\
\hline Service & The service used is specified. \\
\hline Version & Request version. \\
\hline Request & Request name (GetMap). \\
\hline Srs & Spatial Reference System. \\
\hline Format & Output format of map. \\
\hline Bbox & $\begin{array}{l}\text { Bounding box corners (lower left, upper } \\
\text { right) in SRS units. }\end{array}$ \\
\hline Layers & $\begin{array}{l}\text { Comma-separated list of one or more map } \\
\text { layers. Optional if SLD parameter is } \\
\text { present. }\end{array}$ \\
\hline Transparent & Transparency/Opacity is specified. \\
\hline Width & Width in pixels of map picture. \\
\hline Height & Height in pixels of map picture. \\
\hline
\end{tabular}

GetFeatureInfo is an optional function. Not all WMS servers can answer this request. The GetFeatureInfo operation requests the spatial and attribute data for the features at a given location on a map (Figure 4). In this case, the client queries the image and sends the coordinate information and layer of detail to the server (Emem O., 2007) 


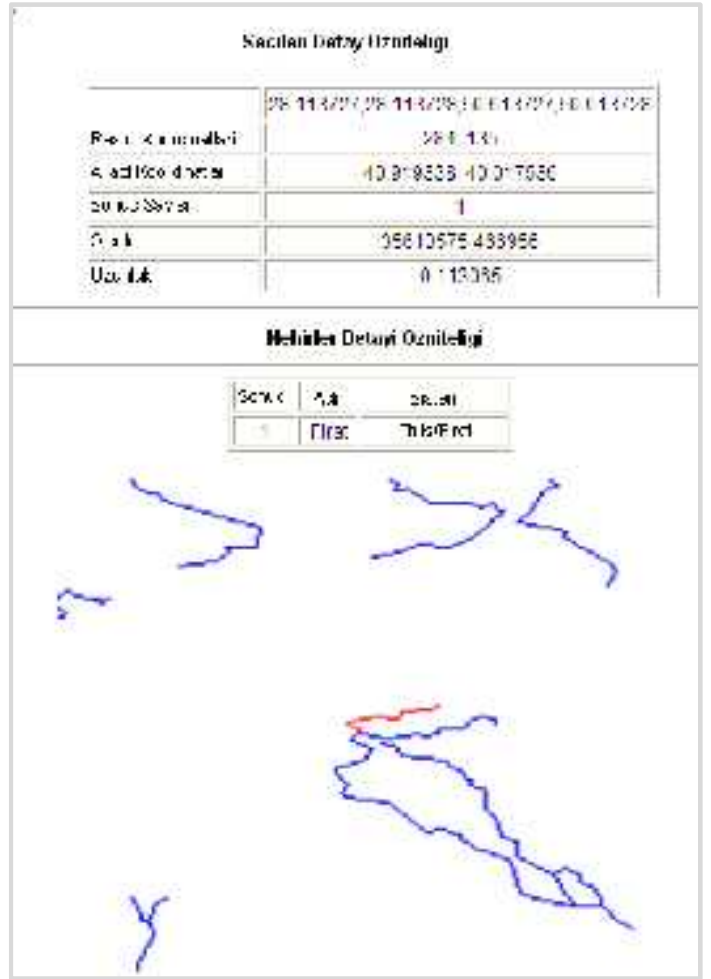

Figure 4. WMS GetFeature request response

WMS has been identified in two types in the OGC document. These are basic WMS and Cascading Map Server. Basic WMS supports the basic functions listed above. The Cascading Server behaves like a client of another WMS and can aggregate images from different servers into a service. It also supports format and coordinate conversions (Emem O., 2007).

\section{WEB FEATURE SERVICE - WFS}

The OGC Web Feature Service (WFS), takes the next logical step of by defining interfaces for data access and manipulation operations on geographic features using HTTP as the distributed computing platform. As it is known WMS interface return only an image, which end-users cannot edit or spatially analyze.

Via these interfaces, a web user or service can combine, use and manage geodata -the feature information behind a map image- from different sources by invoking the following WFS operations on geographic features and elements:

- Create a new feature instance,

- Delete a feature instance,

- Update a feature instance,

- Lock a feature instance,

- Get or query features based on spatial and nonspatial constraints (Open Geospatial Consortium, 2017b).

In this context, WFS; the client generates the request and posts it to a web feature server, the request naturally requests a data transfer from the server or a query on the data held in the server returns the desired result.

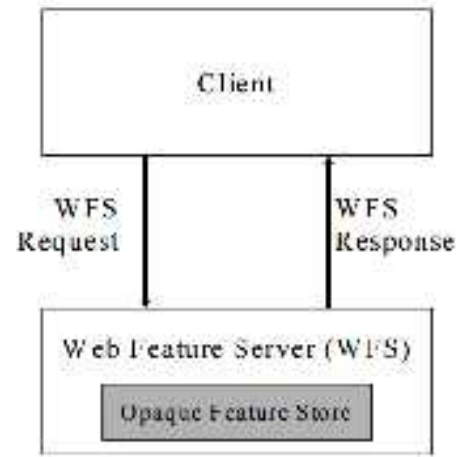

Figure 5. Web Feature Service Client/Server Schema

Requests are generated by the client computer in the direction of the user's request as specified in the WFS standard and are requested from the server with the help of the widely used HTTP protocol. The WFS server examines incoming requests, evaluates the request if the arrived WFS request is appropriate, and sends the final result to the client computer in a format that conforms to the WFS standard. When the WFS has completed processing the request, a query result is generated by either compiling the vector data or the vector data (Figure 5).

After this simple identification, the OGC WFS service service can be further elaborated. The OGC WFS service uses GML (Geography Markup Language) to transfer data and query results. Therefore, the data transferred from the server and sent to the server must be kept absolutely in the GML structure. These transfers do not use industry standard or other popular vector data formats. The GML structure allows a client to request more than one service, and allows a server to serve more than one client. The minimum requirements for a typical WFS service can be listed as follows:

- The Interfaces must be defined in XML (Extensible Markup Language).

- GML must be used to express features within the interface.

- At a minimum a WFS must be able to present features using GML.

- The predicate or filter language will be defined in XML and be derived from CQL as defined in the OpenGIS Catalogue Interface Implementation Specification.

- The datastore used to store geographic features should be opaque to client applications and their only view of the data should be through the WFS interface.

\subsection{WFS Operations}

The details of the GetCapabilities,

DescribeFeatureType and GetFeature functions from the Basic WFS functions and Transaction and LockFeature functions mentioned above are as follows.

\subsubsection{GetCapabilities}

A web feature service must be able to describe its capabilities. Specifically, it must indicate which feature types it can service and what operations are supported on each feature type. 


\subsubsection{DescribeFeatureType}

With this operation, the structure of the vector data presented on the server can be queried. Upon request, it provides information on the structure of any detail type. For example; the feature attribute and geometry's detail is acquired by this capability.

\subsubsection{GetFeature}

This function allows the client to specify what properties the server wants and what data it wants. So it brings back specimen instances. For example; The server can be queried about Turkey's provinces districts in the Mediterranean Region which have population more than 50.000 .

\subsubsection{Transaction}

Through this function, the operation performed on the data is defined. These operations can be generally classified as adding data, changing or deleting the existing data.

\subsubsection{LockFeature}

This function is very important, it allows one or more copies of the processed data to be locked and stored. A LockFeature operation provides a long-term feature locking mechanism to ensure consistency in edit transactions. If one client fetches a feature and makes some changes before submitting it back to the WFS, locks prevent other clients from making any changes to the same feature, ensuring a transaction that can be serialized. If a WFS server supports this operation, it will be reported in the server's GetCapabilities response.

\section{FREE/OPEN SOURCE SERVER/CLIENT SOFTWARES}

\subsection{Server Softwares}

\subsubsection{MapServer For Windows}

MapServer is an Open Source platform for publishing spatial data and interactive mapping applications to the web. Originally developed in the mid-1990's at the University of Minnesota, MapServer is released under an MIT-style license, and runs on all major platforms (Windows, Linux, Mac OS X). All source code is openly available via GitHub.

The biggest advantage of MapServer software is that it supports many OGC standards. While most open source software supports OGC WMS, WFS, WCS, GML standards, MapServer supports OGC WMC, Styled Layer Descriptor -SLD, Sensor Observation Service SOS, Observations and Measurements - OM, Sensor Web Enablement - SWE standards as well.

- Advanced cartographic output

o Scale dependent feature drawing and application execution o Feature labeling including label collision mediation

o Fully customizable, template driven output o TrueType fonts

o Map element automation (scalebar, reference map, and legend)

o Thematic mapping using logical- or regular expression-based classes

- Support for popular scripting and development environments o PHP, Python, Perl, Ruby, Java, and .NET

- A multitude of raster and vector data formats ○ TIFF/GeoTIFF, NetCDF, MrSID, ECW, and many others via GDAL

o ESRI shapfiles, PostGIS, SpatiaLite, ESRI ArcSDE, Oracle Spatial, MySQL

- Map projection support

$\circ$ On-the-fly map projection with 1000s of projections through the PROJ.4 library

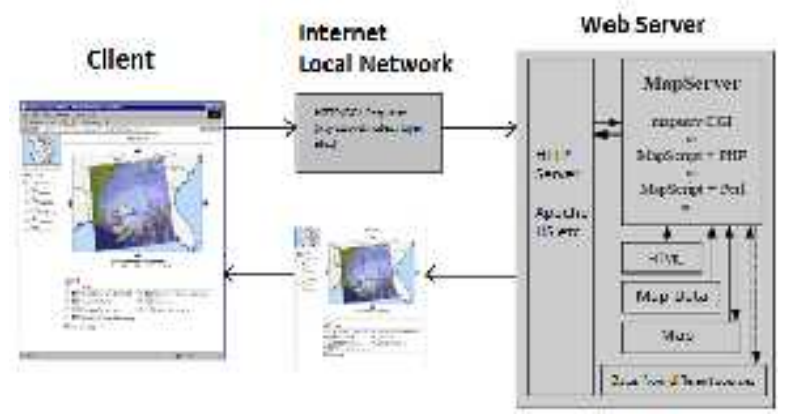

Figure 6. MapServer architecture

For more information visit http://www.mapserver.org.

\subsubsection{GeoServer}

GeoServer is a Java-based software server that allows users to view, share and edit geospatial data. GeoServer is free software. This significantly lowers the financial barrier to entry when compared to traditional GIS products. In addition, not only is it available free of charge, it is also open source. It is already implementing the WMS, WFS and WCS standarts. One of its main strength is the full implementation of the WFS-T protocol. The software supports JPEG, PNG, SVG, GIF, GeoJSON, PDF, GeoRSS, KML / KMZ, GML, Shapefile data formats used in GIS applications.

GeoServer can display data on any of the popular mapping applications such as Google Maps, Google Earth, Yahoo Maps, and Microsoft Virtual Earth. In addition, GeoServer can connect with traditional GIS architectures such as ESRI ArcGIS.

Other geographic data can easily adapt to Geoserver. It is a free software that can work with PostGIS, Oracle Spatial, ArcSDE, DB2, MySQL, Shapefile, GeoTIFF, ECW environments as data source. Geoserver architecture is as shown in Figure 7. 


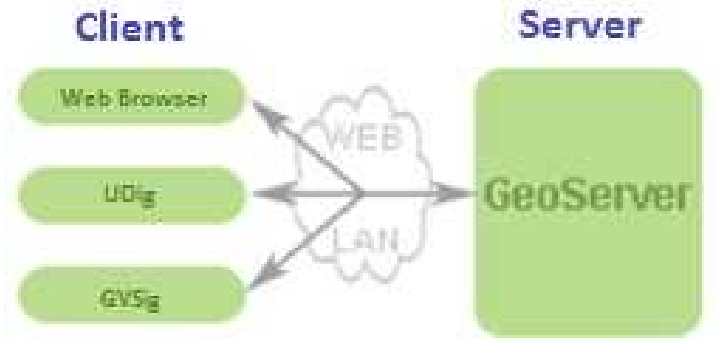

Figure 7. Geoserver architecture

For more information visit http://geoserver.org/

\subsection{Client Softwares}

\subsubsection{Cesium}

Cesium is an open-source JavaScript library for worldclass 3D globes and maps. Cesium was founded by Analytical Graphics, Inc. (AGI) in 2011 as a crossplatform virtual globe for dynamic-data visualization in the space and defense industries. With Cesium, the newest web-based virtual globe application, users have the ability to view spatial data independently of the operating system. OGC WMS, WMTS, CityGML, KML standards are supported.

Cesium;

- Multiple view modes include 2D, 3D and 2.5D Columbus view,

- Stand-alone or local network operations,

- Three-dimensional display no need to install any extension,

- Runs on Windows, Linux, and Mac operating systems,

- There are many features such as dynamic time data display without writing any code.

- Supports visualizing 3D models using gITF

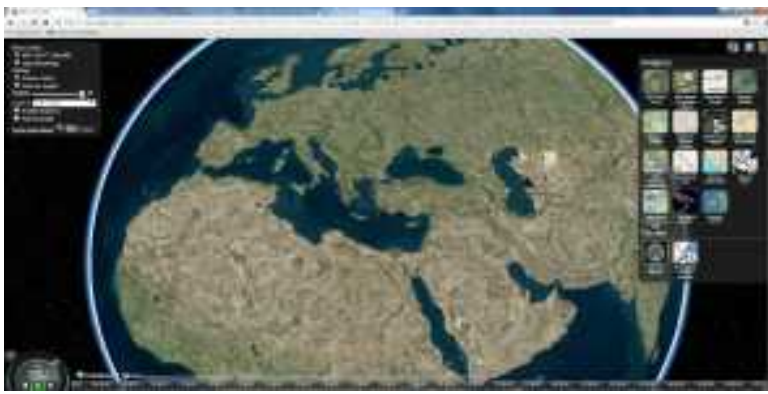

Figure 8. An overview of Cesium

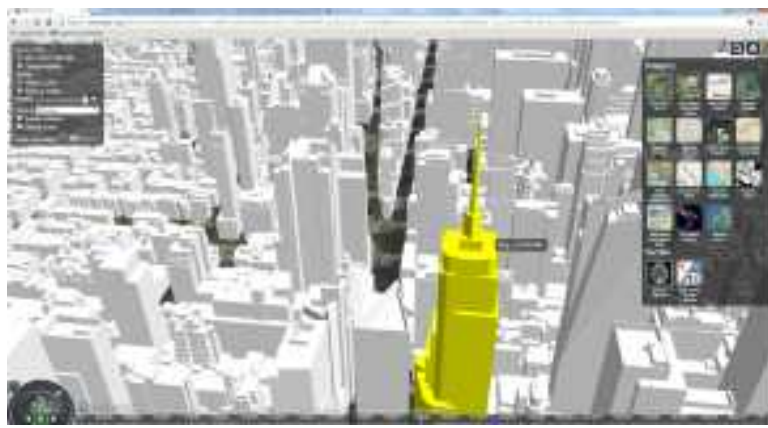

Figure 9. 3D tiles from Cesium
For more information visit http://cesiumjs.org/.

\subsubsection{ArcGIS Explorer}

ArcGIS Explorer is a free viewer software developed by ESRI (Figure 10). With this software;

- Datas can be viewed in Shapefile, KML / KMZ, GPX and raster formats (JPEG 2000, GeoTIFF, MrSID),

- Access to mapping services ArcGIS for Server; ArcIMS; and OGC WMS, and GeoRSS feeds.

- In addition to the maps provided by ArcGIS Online as base images, different sources can be used.

- View maps and globes in any projection or coordinate system.

One of the biggest advantages of the software is that the data can be displayed in both two and three dimensions.

It is also possible for users to develop and add additional tools with free downloadable software development kit (SDK). Users able to access different abilities from ESRI users forums by integrating the extensions that different users have developed.

And different languages are supported on the application (English, French, Japanese, Chinese, German, or Spanish).

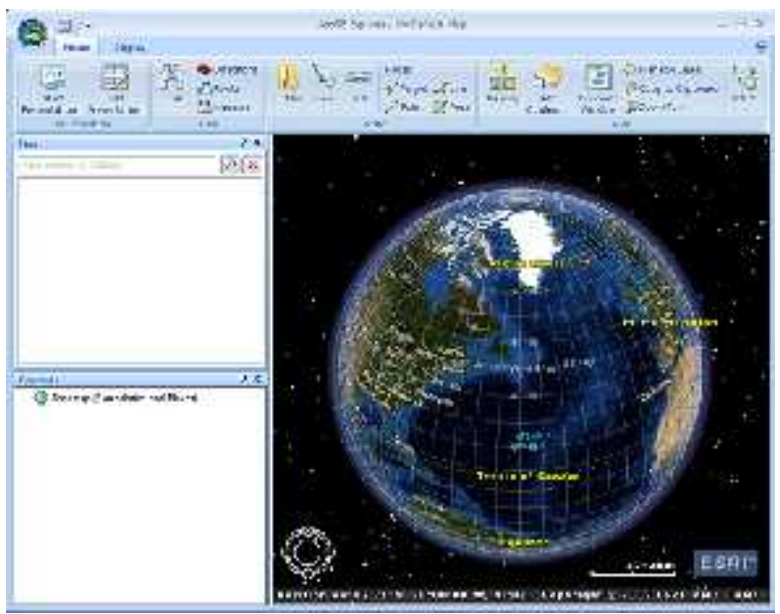

Figure 10. ArcGIS Explorer screen capture

For more information visit http://www.esri.com/software/ arcgis/explorer-desktop.

\subsubsection{Gaia}

Gaia is a platform designed for advanced geospatial network and SDI needs. Based on the CarbonTools PRO open-geospatial development toolkit, this viewer can access an array of geospatial sources such as the OGC WMS, WMTS, WCS, WFS, and FES, services such as Microsoft Bing Maps, Yahoo! Maps and OpenStreetMap (OSM), as well as file formats such as ESRI Shapefiles, Google Earth KML/KMZ, DXF, MIF, GML and GML Simple Features (GMLsf). 
With Gaia users can use geospatial content from different sources and overlay them into a single map view, with each layer individually configured and styled. The Gaia multi-layer view allows seamless use of multiple layers of different types. Panning, zooming and other mapping tools provide a fast and convenient tool for browsing the map. Gaia uses dynamic caching of content to memory, providing enhanced mapping performance.

Gaia includes an open API to allow developers to modify and enhance the application. Gaia makes it easy to create meaningful maps, and includes tools to create your own map symbols. Gaia can run on any compliant Linux and Mac OS platforms.

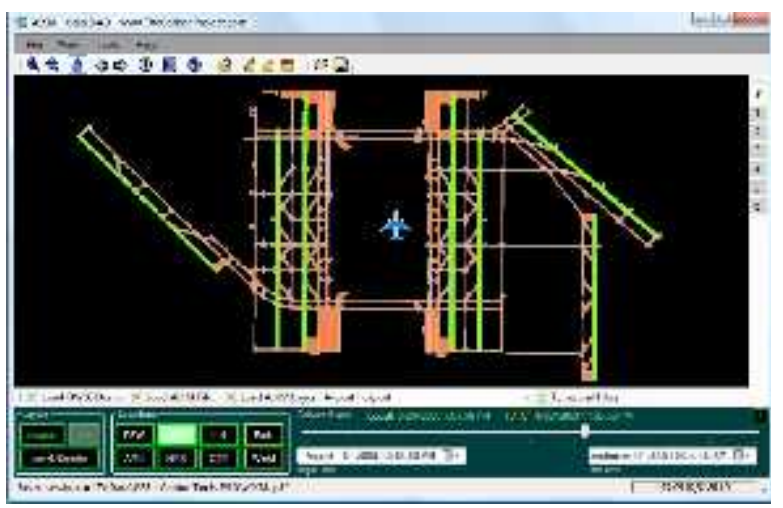

Şekil 11. Gaia screen capture

For more information visit http://www.thecarbonproject.com/ Products/Gaia.

\subsubsection{OpenLayers}

OpenLayers, another open source client software, makes it easy to put a dynamic map in any web page. It can display map tiles, vector data and markers loaded from any source. OpenLayers has been developed to further the use of geographic information of all kinds. It is completely free, Open Source JavaScript, released under the 2-clause BSD License (also known as the FreeBSD).

\section{With OpenLayers;}

- Many map controls will arrive ready for their abilities,

- Render vector data from GeoJSON, TopoJSON, KML, GML, Mapbox vector tiles, and other formats,

- Leverage Canvas 2D, WebGL, and all the latest greatness from HTML5,

- Pull tiles from OSM, Bing, MapBox, Stamen, and any other source. OGC WMS, WFS and untiled layers also supported.

- It will benefit from many user experiences by reaching large participating user forums.

It is easier to integrate GeoServer users with OpenLayers. Figure 13 shows a WFS image of Openlayers.

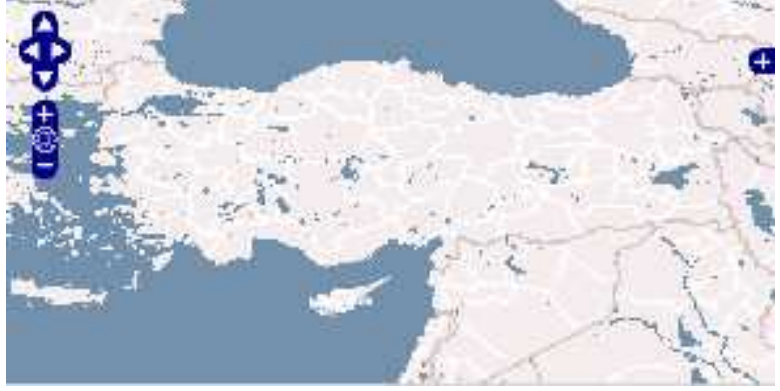

Figure 13. WFS image of Openlayers

For more information visit https://openlayers.org/.

\subsubsection{QGIS}

QGIS is an Open Source Geographic Information System client application. QGIS currently runs on most Unix platforms, Windows, and OS X. QGIS is developed using the Qt toolkit and $\mathrm{C}++$.

The software supports Shapefile, GeoDatabase, MapInfo, Microstation, DXF, MsSQL database formats. Spatially-enabled tables and views using PostGIS, SpatiaLite and MS SQL Spatial, Oracle Spatial, vector formats supported by the installed OGR library, including ESRI shapefiles, MapInfo, SDTS, GML and many more. Online spatial data served as OGC Web Services, including WMS, WMTS, WCS, WFS, and WFS-T. Raster and imagery formats supported by the installed GDAL (Geospatial Data Abstraction Library) library, such as GeoTIFF, ERDAS IMG, ArcInfo ASCII GRID, JPEG, PNG and many more.

\section{With QGIS;}

- Quick view of layers served from different sources,

- Easy-to-use tools,

- Supported 2700 known coordinate systems and user defined coordinate system support,

- Extension support for many analyzes,

- Raster image analysis,

- Possibility of three dimensional display with Qgis2threejs plugin,

- Temporal data display with TimeManager plugin,

- Able to apply topology rules. 


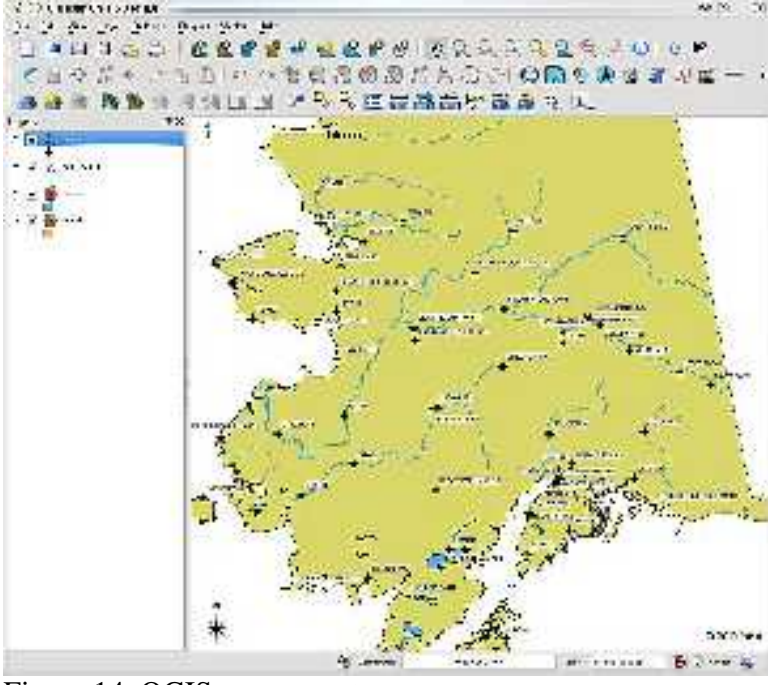

Figure 14. QGIS screen capture

For more information visit http://www.qgis.org.

\subsubsection{GvSIG}

GvSIG is an open source geographic information client software designed to manage geospatial data (Figure 15). It is easy to work in a variety of formats with gvSIG Desktop, vector and raster files, databases and remote services. GvSIG supports OGC WMS, WFS, WCS, WFS-T and WPS standards. GvSIG can access PostGIS, MySQL, ArcSDE, Oracle, JDBC, and CSV databases.

The possibilities provided by GvSIG software;

- Standard GIS tools (zoom in, zoom out, etc.)

- Query with SQL statement,

- Three-dimensional imaging,

- Applying topology rules,

- Raster and vector data analysis,

- Mobile application support.

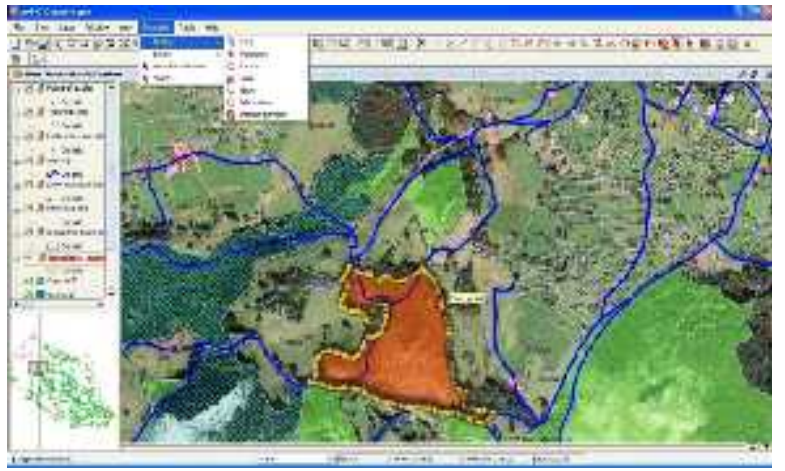

Figure 15. GVSIG scree capture

For more information visit http://www.gvsig.com.

\section{CONCLUSION AND RECOMMENDATIONS}

Today, the importance of communication and information sharing is increasing rapidly. As a consequence of the fact that most of the data used in the studies are spatial data, the importance of sharing these data with other institutions that need it has emerged.
OGC standards play important role in sharing these data according to the principle of interoperability.

In the study, applications that support the OGC web services standards has been examined in the serve and view of vector and raster data. Free and open source software that are under review will also support Individuals/institutions/businesses beyond the budgetary obstacles they face when collecting and serving spatial data. You'll appreciate the ability to be able to freely experiment with technologies without paying any royalties, if you're starting a small company, a private venture, or even a project within a large company.

Publishing of data from any major spatial data source with free/open source software platforms and using OGC standards will serve all users who need the same data. By sharing the spatial data, repeated data collection is prevented and data collection costs will be reduced. By means of the standards, the data obtained from different sources can be easily coordinated, edged and served as images. When your data is kept in open formats, converting from one data type to another is straight forward, and there is probably a piece of software that does exactly that. Custom projects or analyses for specific purposes and users can be easily created and visual presentations are predicted to become feasible. Integration of spatial data from different softwares and data providers could be done without extra software, hardware and geographic transaction costs.

\section{ACKNOWLEDGEMENTS}

This study is derived from the postgraduate thesis that prepared by Eng.Mesud Behlül VAROL who is studying at Selcuk University Institute of Natural Science - Geomatics Engineering Department.

\section{REFERENCES}

Alameh N., 2010, OGC Web Services TestbedOWS-7, AIXM/WXXM Conference Washington DC

Anonim, 2012. E-dönüşüm Türkiye Projesi Birlikte Çalışabilirlik Esasları Rehberi Sürüm 2.1

Anonim, 2016. ISO/TC 211- 1, 2001, ISO 19101 Reference Model, ISO Standartları

AtlasStyler

http://www.geopublishing.org/gp_stable/as.jnlp [Accessed 06 May 2016]

ArcGIS Explorer, http://www.esri.com/software/arcgis/ explorer/index.html [Accessed 19 Apr 2016]

Emem O., 2007. Mekânsal Veri ve Bilgi Altyapısının Uygulamalı Olarak Geliştirilmesi adlı Doktora Tez Çalışması Bildirisi, KTÜ

Europa,

http://ec.europa.eu/idabc/en/document/2319/5644 .html [Accessed 21 Apr 2016] 
Gaia http://www.thecarbonproject.com [Accessed 03 May 2016]

GeoServer, http://geoserver.org [Accessed 19 Apr 2016]

GRASS GIS, http://grass.osgeo.org [Accessed 03 May 2016]

INSPIRE Internet Sitesi, http://inspire.jrc.ec.europa.eu [Accessed 06 Mar 2016]

ISO/TC 211 Web Site [Online], www.isotc211.org [Accessed 19 Apr 2016]

MapServer, http://mapserver.org [Accessed 19 Apr 2016]

Nebert D., Carl REED, Roland M. WAGNER, 2007, Proposal for a Spatial Data Infrastructure Standards Suite: SDI 1.0

Open Geospatial Consortium, 2017a. OGC, http://www.opengeospatial.org/ogc/faq\#5 [Accessed 04 Jan 2017]

Open Geospatial Consortium, 2017b. Standarts, http://www.opengeospatial.org/standards/wfs [Accessed 05 Jan 2017]

Open Geospatial Consortium, 2016. OGC, http://www. opengeospatial.org [Accessed 05 Mar 2016]

OSGeo4W, http://trac.osgeo.org/osgeo4w [Accessed 19 Apr 2016]

OpenGIS, http://www.opengis.ch [Accessed 06 May 2016]

OpenLayers, http://openlayers.org [Accessed 03 May 2016]

Portal gvSIG, http://www.gvsig.com [Accessed 06 May 2016]

Türkiye Ulusal Coğrafi Bilgi Altyapısı Kurulumu Projesi(KYM-75) Fizibilite Etüdü Raporu, 2010. M. Osman ÖZDEM R,

http://www.turksatglobe.com.tr/Views/Projects/

Contents/Files/CbsA/B_010_SUNUM_TASLAK_STA

NDARTLAR_OO.pdf [Accessed 02 Mar 2016]

Quantum GIS, http://www.qgis.org [Accessed 03 May 2016]

uDig, http://udig.refractions.net/ [Accessed 06 May 2016]

Vretanos P. A.,WFS Implementation Specification, https://portal.opengeospatial.org/files/?artifact_id=7176 [Accessed 06 Mar 2016]

Web Map Tile Service, http://www.cubewerx.com/ technology/wmts/ [Accessed 06 Apr 2016]
Copyright (C) International Journal of Engineering and Geosciences (IJEG). All rights reserved, including the making of copies unless permission is obtained from the copyright proprietors. 